\title{
Prospective study of patients with altered mental status: clinical features and outcome
}

\author{
Lim Beng Leong • Kenneth Heng Wei Jian • \\ Alicia Vasu • Eillyne Seow
}

Received: 29 June 2008 / Accepted: 20 July 2008 / Published online: 24 September 2008

(C) Springer-Verlag London Ltd 2008

\begin{abstract}
Aims Patients with altered mental status (AMS) present commonly to the Emergency Department (ED). The aim of this prospective study is to identify the various clinical features of this diverse group of patients and trace their outcomes. This will allow clinicians to be aware of the natural history of the symptom complex and the difficulties in managing them.

Methods In this prospective observational study, we recruited patients aged 18 and above diagnosed with AMS at Tan Tock Seng Hospital ED from December 2006 to October 2007. This is an urban ED. Demographic, clinical, radiology, laboratory data, final diagnosis and length of stay were collected from their entry into the ED till discharge or demise using the ED's and inpatient electronic records.

Results From December 2006 to October 2007, 967 patients with a mean age of 66.5 years diagnosed with AMS were recruited into our study. The total number of CT
\end{abstract}

The views expressed in this paper are those of the author(s) and not those of the editors, editorial board or publisher.

\footnotetext{
L. B. Leong $(\bowtie) \cdot$ K. H. W. Jian $\cdot$ E. Seow

Tan Tock Seng Emergency Department,

11, Jalan Tan Tock Seng,

Singapore 308433, Singapore

e-mail: Beng_Leong_Lim@ttsh.com.sg

K. H. W. Jian

e-mail: khengwj@gmail.com

E. Seow

e-mail: eillyne_seow@ttsh.com.sg
}

\section{A. Vasu}

Biomedical Sciences,

Singapore, Singapore

e-mail: alicia.vasu@gmail.com scans done during the study period was 674, of which 246 (37\%) were abnormal and $428(63 \%)$ were normal. The mean hospital length of stay was 11.6 days. Patients with abnormal CT results stayed longer than those whose results were normal (median of 9 days versus median of 6 days). The three most common causes of AMS in our study population were of neurological (34.4\%), infectious $(18.3 \%)$ and metabolic (12\%) aetiologies. Overall, 106 (11\%) patients died during hospitalisation; 36 (33.9\%) and $39(36.8 \%)$ deaths were attributed to ischaemic stroke and haemorrhagic stroke, respectively.

Conclusions AMS remains a symptom complex that carries a significant length of hospital stay and mortality. The most common causes of AMS are those that require timely intervention and are highly treatable. This study will provide insight into proper allocation of resources to manage this group of patients, from triaging to investigations and treatment at the ED and inpatient levels.

Keywords Altered mental status · Outcome

\section{Introduction}

Altered mental status (AMS) is a symptom complex that continually poses a great challenge in the Emergency Department (ED). It has various causes and treatments, many of which require timely intervention [1].

The purpose of the study is to provide insight into the clinical, demographical features as well as the outcome of patients presenting to our ED through to their entire length of stay in the hospital.

We aim to provide clinicians with the necessary information regarding the epidemiology of this symptom 
complex in an urban ED. This will guide future allocation of resources in the most cost-effective manner in the management of patients with AMS.

\section{Methods}

A prospective observational study was conducted on all patients presenting to the ED with altered mental state (AMS) from December 2006 to October 2007 at Tan Tock Seng Hospital (TTSH), Singapore. TTSH is an urban ED with an annual census of 154,000 in 2007 and receives patients from all regions in the country.

A pilot study using retrospective data in our department together with review of the literature [2-4] formed the basis of an AMS data collection form that was prospectively completed by the ED doctors for all recruited patients.

AMS was defined as a state of drowsiness, unresponsiveness, sudden behavioural change, disorientation or confusion, agitation or hallucination.

All patients above 18 years of age who presented with AMS at triage in the ED were included in the study. Patients referred from another healthcare facility for whom their referring physicians had already worked out the cause for AMS were excluded from the study. Similarly, patients who had sustained major trauma from motor vehicle crashes or falls from heights were excluded. This study was approved by the National Healthcare Group Research Ethics Committee.

Patients were managed by attending emergency physicians (EPs) and ward specialists. The management and the utilisation of specific investigations like computer tomographic (CT) scans were based on the discretion of the attending doctors.

Demographic, clinical, radiological and laboratory data of each patient throughout the stay in the hospital from entry into the ED till discharge or demise were collected. ED electronic notes, AMS forms, inpatient medical records, radiology films and reports were reviewed.

All statistical analyses were performed using SPSS 13.0 (SPSS Inc., IL). All tests were conducted at the 5\% level of significance, with odds ratios (OR) and corresponding 95\% confidence intervals $(\mathrm{CI})$ reported where applicable.

\section{Results}

From December 2006 to October 2007, 967 patients with AMS were recruited into our study. The annual attendance for 2007 was 154,000 . Table 1 describes the baseline demographic data for the patients recruited. The majority of patients $(60 \%)$ were 65 years and above. AMS patients presented most commonly (67\%) within the first $24 \mathrm{~h}$ of
Table 1 Demographics of study population $(\mathrm{N}=967)$

\begin{tabular}{|c|c|}
\hline Variables & Total \\
\hline \multicolumn{2}{|l|}{ Gender } \\
\hline Males & $459(48 \%)$ \\
\hline \multicolumn{2}{|l|}{ Ethnicity } \\
\hline Chinese & $747(77 \%)$ \\
\hline Malay & $106(11 \%)$ \\
\hline Indian & $86(9 \%)$ \\
\hline Others & $28(3 \%)$ \\
\hline \multicolumn{2}{|l|}{ Age range (in years) } \\
\hline 65 and above & $581(60 \%)$ \\
\hline \multicolumn{2}{|l|}{ Type of AMS } \\
\hline Drowsiness & $331(51 \%)$ \\
\hline Sudden behavioural change & $180(27 \%)$ \\
\hline Unresponsiveness & $248(37 \%)$ \\
\hline Disorientated/confused & $199(30 \%)$ \\
\hline Agitation & $51(8 \%)$ \\
\hline Hallucination & $41(6 \%)$ \\
\hline \multicolumn{2}{|l|}{ Duration of AMS } \\
\hline Acute $(24 \mathrm{~h})$ & $647(67 \%)$ \\
\hline Subacute (less than 7 days) & $215(22 \%)$ \\
\hline Chronic (more than 7 days) & $105(11 \%)$ \\
\hline \multicolumn{2}{|l|}{ Concurrent medications } \\
\hline Benzodiazepine & $55(6 \%)$ \\
\hline Tricyclic anti-depressant & $8(1 \%)$ \\
\hline SSRIs & $54(6 \%)$ \\
\hline Anti-psychotics & $66(7 \%)$ \\
\hline Anti-coagulant & $34(4 \%)$ \\
\hline Anti-platelet & $121(13 \%)$ \\
\hline Alcohol & $53(6 \%)$ \\
\hline
\end{tabular}

onset of symptom with drowsiness as the most common presenting complaint (51\%).

The frequency of AMS increases with age in a nonGaussian manner with a mode of 75 years and mean of 66.5 years (SD of 18.1).

After the initial screening investigations of an electrocardiogram and capillary blood sugar, the subsequent management was based on the discretion of the attending emergency physician or inpatient specialists. Of note, 674 patients $(70 \%)$ received CT scan of the brain, of which 394 $(58 \%)$ were performed at the ED and the rest at the ward level. Of all CT scans performed, 246 (36\%) were reported to be abnormal; 135 were found to have ischaemic stroke, 106 to have haemorrhagic stroke and 5 to have tumours.

All patients were admitted to either the medical, neurology or neurosurgical units based on the attending EP's provisional diagnosis.

The various definitive causes of AMS upon discharge or demise are demonstrated in Table 2.

In the younger age group of 16-64 years, the various AMS causes were more evenly distributed with neurological, psychiatric and toxicological aetiologies as relatively 
Table 2 Causes of AMS in patients aged 16-64 $(\mathrm{N}=392)$ and $>65$ $(\mathrm{N}=600)^{*}$

\begin{tabular}{lll}
\hline Cause of death & $18-64$ years & $\begin{array}{l}65 \text { years } \\
\text { and above }\end{array}$ \\
\hline Neurological & $11.7 \%$ & $22.7 \%$ \\
Infectious & $4.3 \%$ & $14.0 \%$ \\
Metabolic & $4.3 \%$ & $7.8 \%$ \\
Toxicological & $6.0 \%$ & $2.2 \%$ \\
Cardiac & $1.5 \%$ & $5.9 \%$ \\
Organ specific impairment & $5.0 \%$ & $1.7 \%$ \\
Psychiatric & $1.1 \%$ & $1.7 \%$ \\
Trauma & $1.0 \%$ & $1.4 \%$ \\
Miscellaneous & $3.2 \%$ & $4.7 \%$ \\
\hline
\end{tabular}

*N>967 as some patients were assessed to have more than one cause of AMS

more common. In the older age group of $>65$ years, neurological, infectious and metabolic aetiologies were more common. In the latter group of patients, the sources of infection include urinary tract infection (36\%), pneumonia $(22 \%)$ and sepsis $(27 \%)$.

The outcomes of these AMS patients were followed up prospectively. The mean hospital length of stay was 11.6 days (median 7 days; range $1-137$ days). Patients with abnormal CT results stayed longer than those whose results were normal (median of 9 days; range 1-137 days compared with median of 6 days; range 1-114 days; $\mathrm{p}=0.001)$.

Overall, 106 (11\%) patients died during hospitalisation; $36(33.9 \%)$ and $39(36.8 \%)$ deaths were attributed to ischaemic stroke and haemorrhagic stroke, respectively.

We investigated the causes of mortality between the younger (18-64 years) and older ( $>65$ years) subgroups. The majority of younger patients died from haemorrhagic stroke, while in older patients, mortality is distributed amongst ischaemic stroke (38\%), haemorrhagic stroke (30\%) and others (chiefly infection; 20\%). This is demonstrated in Table 3.
Table 4 Distribution of outcomes of surviving AMS patients $(\mathrm{N}=861)$

\begin{tabular}{ll}
\hline Outcome & Number \\
\hline Discharge to primary care & 651 \\
Discharge to nursing home & 21 \\
Transfer to rehabilitation hospital & 33 \\
Discharge to hospital outpatient clinic & 107 \\
Discharged against medical advice & 26 \\
Missing data* & 23 \\
\hline
\end{tabular}

* Missing data includes those with incomplete data or those whose disposition was not available at time of conclusion of study

Table 4 illustrates the outcomes of the survivors. The majority of patients were discharged to primary care $(76 \%)$ or the hospital's outpatient clinics (12\%). A small minority of patients were transferred to step-down care comprising of nursing homes $(2.4 \%)$ and rehabilitation hospitals $(3.8 \%)$.

\section{Discussion}

AMS remains a diagnostic challenge in the Emergency Department. It has a multitude of possible aetiologies. Acute onset of AMS within $24 \mathrm{~h}$ is the most common reason for seeking consultation at the ED. Among the various aetiologies, neurological causes are the most common, and our findings agree with what had been reported by Kanich et al. [5]. Futhermore, this has important downstream implications for triaging and costeffective use of investigations, especially $\mathrm{CT}$ scan of the brain $[6,7]$.

We have demonstrated that AMS is a symptom complex that carries a high admission rate, a long length of stay and significant mortality rate (11\% in our study, and this agrees with what is reported by Zeynep Kekec et al. [8]). Our study has also demonstrated that the causes of AMS vary amongst the different age groups. Although it is not as

Table 3 Distribution of causes of mortality between two age subgroups

\begin{tabular}{lllr}
\hline Cause of death & $\begin{array}{l}18-64 \text { years old } \\
(\mathrm{n}=30 ; 28 \%)\end{array}$ & $\begin{array}{l}65 \text { years and above } \\
(\mathrm{n}=76 ; 72 \%)\end{array}$ & $\mathrm{p}$ value \\
\hline Trauma & $1(3.3 \%)$ & $5(6.6 \%)$ & 0.673 \\
Cardiac & $1(3.3 \%)$ & $4(5.3 \%)$ & 1.000 \\
Others* & $5(16.7 \%)$ & $15(19.7 \%)$ & 0.790 \\
Ischaemic stroke & $7(23.3 \%)$ & $29(38.2 \%)$ & 0.176 \\
Haemorrhagic stroke & $16(53.3 \%)$ & $23(30.3 \%)$ & $\mathbf{0 . 0 4 3}$ \\
\hline
\end{tabular}

*Others include infective causes or combination of infective and CVA; trauma includes relatively minor mechanisms like falls 
common as other complaints like chest pain, it is one that challenges the physician's judgment, and time is required in the evaluation of such patients. This also impacts on resource utilisation within the hospital.

AMS will continue to be a diagnostic challenge for EPS worldwide as the world's population ages. Appropriate management of AMS in geriatric patients in the ED requires substantial staff time, training, resources and commitment. In addition, EP's satisfaction in management of this group of patients who require prolonged clinician time will also be affected in this high-stress environment [9].

\section{Conclusion}

AMS is and will be a growing symptom complex that prompts caregivers to consult the public healthcare system particularly in the context of a rapidly aging population in many countries.

The common and important causes of AMS require timely diagnosis, intervention and are largely amenable to treatment. AMS remains a symptom that carries a significant degree of morbidity and mortality, especially in elderly patients with neurological aetiologies.

Future research should be targeted to exploring costeffective ways to triage, investigate and manage this symptom complex.

\section{Conflicts of interest None.}

\section{References}

1. American College of Emergency Physicians (1999) Clinical policy for the initial approach to patients presenting with altered mental status. Ann Emerg Med 33(2):251-281, Feb

2. Naughton BJ, Moran MB, Kadah H et al (1995) Delirium and other cognitive impairment in older adults in an emergency department. Ann Emerg Med 25:751-755

3. Bross MH, Tatum NO (1994) Delirium in the elderly patient. Am Fam Physician 50:1325-1332

4. Kanich W Altered mental status: evaluation and etiology in the ED. Am J Emerg Med 20(7):613-617

5. Kanich et al (2002) Altered mental status: evaluation and etiology in the ED. Am J Emerg Med 20:11

6. Sinclair DE, Kovacs G, Hillis M, Cranial CT (1993) Scans: emergency department utilization. J Emerg Med 11:643-646

7. Rothrock SG, Buchanan C, Green SM, Bullard T, Falk J, Langen M (1997) Cranial computed tomography of adult patients without a recent history of head trauma: prospective analysis. Acad Emerg Med 4:654-661

8. Kekec Z et al (2008) Analysis of altered mental status in Turkey. Int J Neurosci 118(5):609-617, May

9. Shumacher JG Emergency medicine and older adults: continuing challenges and opportunities. Am J Emerg Med 23:556-560

I, Dr. Lim Beng Leong, am currently working as an associate consultant in Tan Tock Seng Emergency Department, an urban ED in Singapore. I have done a retrospective study of AMS patients that was used as a pilot for the current study. My other research interests are in emergency cardiac care, and I am currently working on projects of pre-hospital ECGs and their impact on mortality and baseline ECG features that are important to recognize at triage, such as Wellen's and Brugada ECGs. 